\title{
Diagnóstico del desempeño docente en el esquema de evaluación de asesores de los Programas Educativos del Sistema a Distancia en la UAEM
}

\section{Diagnostic of teaching performance in the evaluation scheme of advisors of the Education Programs of the Distance System in the UAEM}

\author{
HUERTA-XINGÚ, Francisco $\dagger^{*}$, ALVARADO-CAMPUZANO, Verónica y HERNANDEZ-SUAREZ, \\ Alejandro
}

\begin{abstract}
Universidad Autónoma del Estado de México. Dirección de Educación Continua y a Distancia.Instituto Literario 100, Centro, 50000 Toluca de Lerdo, Méx.
\end{abstract}

\author{
ID $1^{\mathrm{er}}$ Autor: Francisco, Huerta-Xingú / ORC ID: 0000-0001-9838-7888 \\ ID $1^{\text {er }}$ Coautor: Verónica, Alvarado-Campuzano / ORC ID: 0000-0003-0096-8938 \\ ID $2^{\circ}$ Coautor: Alejandro, Hernandez-Suarez / ORC ID: 0000-0003-3958-5272
}

\begin{abstract}
Resumen
La presente investigación tiene como objetivo generar un diagnóstico del desempeño docente en la Universidad Autónoma del Estado de México, derivado de los resultados en una encuesta de satisfacción aplicada a los alumnos en la que se identifica que están parcialmente de acuerdo en establecer una comunicación adecuada entre los integrantes de la comunidad correspondiente a modalidad no escolarizada, sistema a distancia. Se revisan tres vertientes: los tiempos de espera en la evaluación de las actividades, los tiempos en la atención de peticiones académicas y la cantidad de publicaciones académicas. El tipo de investigación es cuantitativa y la obtención de datos es directamente de los reportes obtenidos de la plataforma educativa utilizada en la Universidad Autónoma del Estado de México; en la investigación se analizan con técnicas estadísticas. Como contribución se proponen estrategias a implementarse de manera que coadyuven a establecer una adecuada comunicación entre los integrantes del grupo con miras a cambiar la percepción estudiantil, alineado hacia una mayor credibilidad en la modalidad.
\end{abstract}

Comunicación en la Educación a distancia, Desempeño docente, Percepción estudiantil

\begin{abstract}
The objective of this research is generate a diagnostic of teaching performance at the Autonomous University of the State of Mexico, derived from the results in a satisfaction survey applied to students in which it is identified that they partially agree to establish adequate communication between the members of the community corresponding to non-school modality, distance system. Three aspects are reviewed: the waiting time in the evaluation of the activities, the time in the attention of academic requests and the quantity of academic publications. The type of research is quantitative and data collection is directly from the reports obtained from the educational platform used at the Autonomous University of the State of Mexico; research analyzed data with statistical techniques. As a contribution, proposed implement strategies in a way that helps to establish adequate communication between the members of the group with a tendency to changing student perception, aligned with greater credibility in the modality.
\end{abstract}

Communication in virtual education, Teacher performance, Student perception

Citación: HUERTA-XINGÚ, Francisco, ALVARADO-CAMPUZANO, Verónica y HERNANDEZ-SUAREZ, Alejandro. Diagnóstico del desempeño docente en el esquema de evaluación de asesores de los Programas Educativos del Sistema a Distancia en la UAEM. Revista de Políticas Universitarias. 2019. 3-8: 23-32.

\footnotetext{
* Correspondencia del Autor (Correo electrónico: valvarado@uaemex.mx)

$\dagger$ Investigador contribuyendo como primer autor.
} 


\section{Introducción}

El principal sostén de cualquier institución son las personas, especialmente de una con arraigada tradición progresista como la UAEM, cuyo objeto es "generar, estudiar, preservar, transmitir y extender el conocimiento universal y estar al servicio de la sociedad"; y por fines "impartir educación media superior y superior, llevar a cabo la investigación humanística, científica y tecnológica; difundir y extender los avances del humanismo, la ciencia, la tecnología, el arte y otras manifestaciones de la cultura" (Universidad Autónoma del Estado de México, 2007). Para cumplir de manera pertinente con lo que nos demanda la sociedad y con las recomendaciones de organismos externos evaluadores de la calidad en cuestiones educativas (COPEEMS, COPAES, CIEES, PNPC), los procesos de enseñanza-aprendizaje en la UAEM pueden ser administrados en modalidades educativas no escolarizada y mixta. Ambas modalidades, para llevarse a cabo, requieren la mediación de la tecnología.

La docencia es la función sustantiva de la Universidad pública donde se evidencia la creciente demanda de enseñanza media superior y superior, cuestión que constituye el mayor desafío institucional y exige imaginación, voluntad y recursos financieros para cumplir una encomienda histórica.

\section{Política}

- Impulsar una docencia de calidad basada en la capacitación, actualización y formación permanente del personal académico que se relaciona cotidianamente con los estudiantes en las aulas.

- Diseñar entornos de aprendizaje orientados a la innovación y al pensamiento reflexivo de docentes y alumnos.

\section{Estrategias.}

- Mejorar la formación de docentes y estudiantes mediante entornos virtuales de aprendizaje.

- Mejorar la calidad de la enseñanza y el aprendizaje guiados por las tendencias y buenas prácticas educativas en el ámbito nacional e internacional.

\section{Líneas de acción.}

Desarrollar modalidades educativas en función a los adelantos de las tecnologías de la comunicación y la información para las opciones: presencial, a distancia, virtual y mixta.

Aunado a lo anterior, la ANUIES indica:

"Aquellas instituciones que deseen innovar con programas educativos a distancia y en sus modalidades no convencionales habrán de prestar atención particular a la identificación de problemas específicos adecuados a la población a la que van dirigidos, así como en establecer metas precisas que involucren a las comunidades académicas, en el mejoramiento de las condiciones de trabajo, desarrollo profesional de sus profesores y en la atención de los estudiantes, para alcanzar una mejor experiencia profesional y formativa." (ANUIES-SINED, 2017)

Por lo cual, se promueve una docencia comprometida y especializada en los diversos programas, niveles y sistemas educativos, profesionalizada y actualizada, capaz de innovar en las tareas de supervisión de la enseñanza y las prácticas escolares.

Para la UAEM, el reto es superar las limitaciones tecnológicas y de infraestructura en un contexto de escasos recursos. El fin es asegurar un acceso más equitativo a los notables avances y modalidades de la enseñanzaaprendizaje.

El empleo de las TIC será un mecanismo generalizado en la universidad, como medio para que nuestros alumnos sean competitivos en conocimientos, capacidades y habilidades propias de su disciplina o grado educativo.

Además, estarán capacitados para "aprender a aprender" con métodos sistemáticamente apoyados en el correcto manejo de las TIC.

De esta manera podrán mantenerse actualizados en un entorno de alta velocidad de generación del conocimiento que caracteriza al mundo contemporáneo (pp. 52-53). 
Por lo anterior, un elemento central de la estrategia en materia educativa será iniciar en la UAEM un proceso claro de transformación para convertirse en una universidad digital, que implicará, entre otras cosas, incorporar las TIC a la docencia, la investigación, la difusión cultural, la extensión y la gestión; utilizar cotidianamente plataformas de software educativo; proporcionar infraestructura tecnológica compartida; disponer de esquemas de educación virtual apoyados en la implantación de sistemas modernos de gestión de contenidos de aprendizaje que incluyan, entre otros componentes repositorios de objetos de aprendizaje (como cursos y conferencias digitales) y herramientas de autor, de publicación y de colaboración, detallados en la fase de especificación técnica del proyecto (p. 53). Como es evidente la función del docente en este ejercicio es central, por esa razón se ha estimado la necesidad de contar con un

\section{Esquema de evaluación de asesores de los PE del Sistema a Distancia.}

El profesor que se incorpora a la modalidad a distancia o mixta cumple diferentes funciones; puede ser elaborador de material didáctico, coordinador, asesor o tutor.

\section{Situación actual}

Durante el último ciclo escolar se ha tenido un reporte fidedigno (a través de las herramientas de seguimiento de SEDUCA) de las actividades que el profesor en el Sistema a distancia ha realizado, con la finalidad de asegurar que los estudiantes transitan de manera pertinente en los procesos de enseñanza-aprendizaje encaminados al logro del perfil de egreso del plan de estudios correspondiente. Con respecto a la selección de los profesores/asesores del Sistema a distancia que se ha venido realizando hasta la fecha podemos mencionar lo que Peter Drucker aclara acerca de que las decisiones relativas a las personas son importantes, pues determinan la capacidad de rendimiento de la organización además de poner en juego el respeto de la misma. Si las decisiones relacionadas con la selección o promoción de personal no son objetivas, transparentes y apegadas a la competencia del personal hay el riesgo de desviar el comportamiento del resto de los recursos humanos (Drucker, 1988). Las demás personas tenderán a comportarse como ven comportarse a quienes son recompensados o ascendidos.
El problema afecta a la institución cuando los beneficios recaen en características que se distancian de las pautas marcadas por la selección, sea tradicional o por competencias, y son más de índole personal o social: halagos, no rendimiento, lealtad a la persona en el poder más que a la institución, favores, simpatías, etc.

Especialmente significativo resulta el comentario de Koontz y Weirich (1998, pág. 93) respecto a la administración mexicana. Él destaca que los ascensos obedecen más al grado de influencia que tiene el superior que a la preparación, conocimientos y habilidades de los individuos. La consecuencia es que la lealtad a las personas en los puestos superiores prevalece por sobre la lealtad a la institución.

Con el propósito de asegurar que los profesores cumplan de manera adecuada con las obligaciones establecidas en el Estatuto Universitario (2007), en el Plan General de Desarrollo 2009-2021, el Plan de trabajo de la Administración (2017 - 2021) y los organismos externos evaluadores y certificadores, se propone el siguiente esquema de evaluación.

La evaluación de la función de asesoría debe concebirse como una acción continua y permanente en los campos de la gestión escolar y la pedagógica. De forma que permita identificar, reconocer y valorar fortalezas $y$ debilidades, con el fin de establecer y desarrollar estrategias que lleven a un mejoramiento continuo. Por lo tanto, la propuesta de evaluación está concebida para realizarse como un proceso sistemático de obtención de información confiable y válida que permita ponderar el logro de los resultados y el grado de cumplimiento de las responsabilidades inherentes a las funciones del asesor.

Las ideas básicas que deben guiar el proceso de evaluación se expresan en unos principios y pautas orientadoras que deben seguir tanto los evaluadores como los evaluados, con el propósito de alcanzar los fines propuestos.

Los principios que rigen la evaluación son: 
Objetividad: la evaluación cumple con el principio de objetividad cuando el proceso identifica diferentes fuentes - personales y documentales - para generar la información y las evidencias, sobre las cuales se basa el juicio de valor acerca del desempeño; utiliza la comparación de información proveniente de diferentes fuentes para encontrar concordancias y resolver discrepancias; prescinde de criterios subjetivos en las valorizaciones asignadas y, determina los procedimientos y las competencias de las instancias que participan en el proceso de evaluación.

Pertinencia: la evaluación cumple este principio cuando responde a los parámetros generales planteados de acuerdo con las funciones y responsabilidades del evaluado y facilita una distribución razonable de las valorizaciones en diferentes posiciones que permite distinguir adecuadamente desempeños inferiores, medios y superiores.

Transparencia: la evaluación es transparente cuando hay un amplio conocimiento de los criterios, instrumentos y procedimientos de evaluación por parte de los involucrados; se acuerda y establece el período de evaluación y, se basa en información cualitativa o cuantitativa, fiablemente sustentada.

Participación: La evaluación cumple con este principio cuando la relación entre el evaluado y evaluador está abierta al diálogo y al consenso; el evaluado se involucra activamente en la planeación y formulación de los objetivos del plan de desarrollo profesional

Equidad: Se cumple este principio cuando se reconoce el contexto y necesidades del evaluado durante el proceso y los criterios generales de evaluación son conocidos por todos los involucrados.

Una vez considerado los principios anteriores, la implementación del proceso de evaluación debe contar con un momento de preparación que garantice:

- Conocimiento total y plena comprensión del proceso general de la evaluación por parte de los asesores académicos y la comunidad educativa.
- Conformación del equipo evaluador para la recolección de información y seguimiento al plan de trabajo de cada asesor.

- Conocimiento de los instrumentos y la metodología por parte del equipo evaluador y los evaluados.

- Definición y concertación de los criterios y los instrumentos necesarios para la recolección de la información, el seguimiento a los compromisos, el registro de las observaciones del asesor, diálogos, reuniones y demás actividades en torno a la evaluación de desempeño y a la implementación del SIRAAE.

En este sentido, los mecanismos utilizados para la evaluación de los asesores deben tomar en cuenta los siguientes aspectos:

- Conversar de manera referenciada con los pares.

- Identificar las necesidades de formación.

- Evaluar de manera objetiva su desempeño profesional.

Esquema de evaluación.

Tomando en cuenta.

1. Identificación de las actividades deseables de los profesores/asesores.

Esta actividad se efectuó en dos momentos, un pilotaje y después, con el apoyo de una encuesta aplicada en línea a estudiantes, profesores y coordinadores de PE a distancia.

2. Comparación y análisis de lo obtenido en la etapa anterior lo que establecen las instancias externas que evalúan la calidad educativa de los programas en sus diferentes niveles: COPAES, CIEES, PNPC y la ANUIES.

3. Elaboración de la descripción y el Perfil de Puesto del profesor/asesor de PE a distancia de cada nivel educativo.

4. Elaboración de los instrumentos de evaluación.

5. Evaluación de los profesores. 


\section{UAEM}

\section{Profesor del Sistema a Distancia de la}

Descripción de puesto

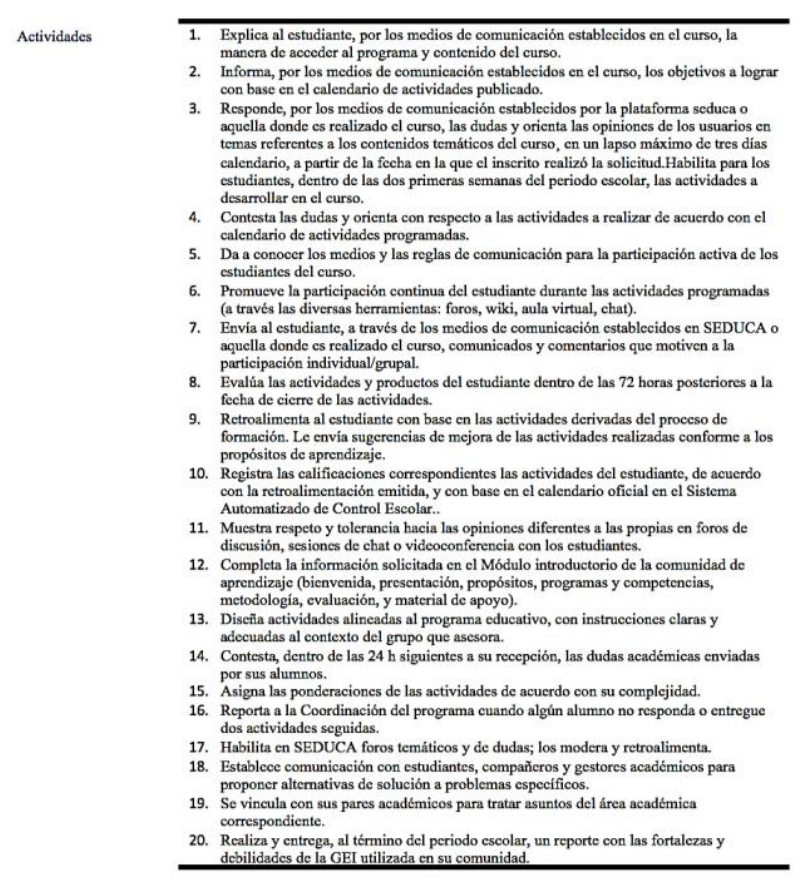

Figura 1

En años recientes, el término de innovación se ha empleado en educación para dar cuenta de la capacidad de cambio y adaptación poseen los diferentes agentes educativos y las propias escuelas, porque esto vendría a ser como una acción planificada con propósitos de transformación y mejoramiento de la calidad educativa, por esta razón entendemos que el cambio educativo como cualquier modificación no evolutiva que se produce en la realidad educativa (Tejada, 1998).

Por ello, en el caso de la innovación educativa se considera que el cambio es la causa y el fin de una innovación, es decir, se innova para generar cambios.

Afirma (Arias, 1998) consideran que la innovación educativa es el estudio de las estrategias o procesos de cambio.
Es importante mencionar que el contexto disciplinario en el que surge la innovación, vinculada al cambio y a la reforma educativa para el mejoramiento en la administración, cuyo marco de referencia es sistémico y se centra en la transformación aparente de la vida escolar de las organizaciones pero desde el marco de la (OCDE, 2001) se entiende como un plan deliberado para mejorar la escuela que tenga un claro inicio y un conjunto identificable de cambios que muevan la escuela hacia una organización de aprendizaje que vayan referidas al currículo, a la enseñanza, al desarrollo del personal y tendrá por objetivo la calidad de la educación, en sus costos o en la equidad de acceso.

Finalmente, podemos agregar que la innovación educativa es un proceso que involucra la selección, organización y utilización creativa de elementos vinculados a la gestión institucional, el currículum y la enseñanza, siendo normal que impacte en más de un ámbito porque suele tener entre otras funciones: Formular estrategias de difusión y promoción de los programas de investigación e innovación educativa; establecer alianzas con comunidades del conocimiento y redes de colaboración al interior de las escuelas y con otras instituciones educativas; supervisar el desarrollo de materiales educativos, así como, promover el desarrollo de proyectos de innovación educativa para la mejora de las prácticas institucionales.

A pesar de las acciones de la reforma educativa, los cambios en la innovación y en el funcionamiento diario de la escuela siguen siendo débiles, e imperceptibles la pregunta es ¿Dónde hacer cambios en la práctica educativa o en la formación? ¿Qué nos está faltando que no se está atendiendo, será acaso la formación del docente?

De acuerdo con la ANUIES: “...Es necesario aclarar la posibilidad de ampliar la cobertura y el acceso al nivel superior por parte de las instituciones educativas, pero al hacerlo cada una deberá priorizar si dentro de su misión y su visión se encuentra el desarrollo de estas modalidades, no sólo designará mecanismos de coordinación, financiamiento y evaluación y certificación para lograrlo, sino también será el marco de referencia de su modelo educativo." (ANUIES-SINED, 2017). 
Por otro lado, la ANUIES indica "Como segunda y tercera acciones principales, se encuentran el desarrollo de modelos pedagógicos (489 puntos) y/o educativos (506 puntos) que sustenten el aprendizaje a distancia...Las ies consideraron que como cuarta acción se deben regular los programas de ESaD (549 puntos)" (ANUIES-SINED, 2017) Por lo cual, es necesario tomar acciones hacia la regulación de los programas.

"Las universidades e Instituciones de Educación Superior que han iniciado el desarrollo de programas educativos a distancia y en sus modalidades no convencionales, lo han hecho con enfoques centrados en la enseñanza y el aprendizaje de los estudiantes, asunto relevante cuando se requiere focalizar la atención en la calidad de los procesos y en resultados concretos." (ANUIES-SINED, 2017) Para focalizar la calidad se requiere de datos fidedignos que muestren un panorama longitudinal sobre el comportamiento de la atención académica a los alumnos, así que para la presente investigación utilizamos los reportes de la plataforma educativa SEDUCA a través de la cual, los alumnos llevan a cabo sus estudios en la modalidad no escolarizada (sistema a distancia).

"Aquellas instituciones que deseen innovar con programas educativos a distancia y en sus modalidades no convencionales habrán de prestar atención particular a la identificación de problemas específicos adecuados a la población a la que van dirigidos, así como en establecer metas precisas que involucren a las comunidades académicas, en el mejoramiento de las condiciones de trabajo, desarrollo profesional de sus profesores y en la atención de los estudiantes, para alcanzar una mejor experiencia profesional y formativa." (ANUIES-SINED, 2017) Como se indica en la Figura 2. Por ello, se pretende con este trabajo identificar alguna problemática con la finalidad de proponer estrategias para provocar un cambio que derive en una mejorar en el proceso de enseñanza-aprendizaje.

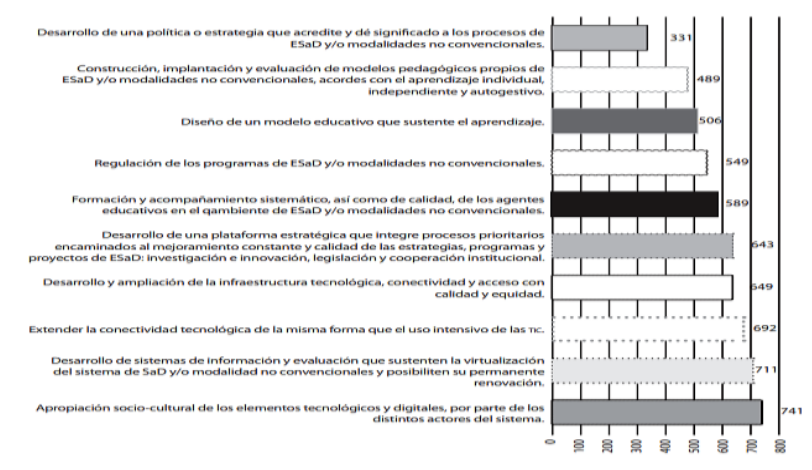

Figura 2 Acciones para asegurar el desarrollo y la calidad de la ESaD (ANUIES-SINED, 2017)

Fuente: ANUIES-SINED, 2017

\section{Tiempos que emplean los docentes para evaluar actividades en modalidad no escolarizada}

El diagnóstico de ESaD en México indica que "el tiempo de respuesta de un profesor a un estudiante se otorga en un lapso de 25 a 48 horas prácticamente, la mitad de las instituciones contestaron que dicha información la tendrían los alumnos en ese periodo; sin embargo, otro grupo importante contestó que esa respuesta de tutores a alumnos la obtendrían en menos de 24 horas (27\%), asunto que resalta por la inmediatez en atender las necesidades de los estudiantes y desplegar recursos para otorgarles alguna respuesta." (ANUIES-SINED, 2017). En este estudio se considera como uno de los parámetros en la evaluación del desempeño docente de modalidad no escolarizada, el tiempo de respuesta en calificar las evidencias. Estrategias para la implementación del esquema de evaluación.

- Sensibilizar a la comunidad universitaria acerca de los beneficios, retos y ventajas de la evaluación de los asesores de los PE en modalidades no escolarizadas y mixtas.

- Elaborar los instrumentos de evaluación.

- Evaluar a los profesores/asesores.

- Analizar los resultados.

- Programar acciones correctivas.

Se envió una encuesta de percepción estudiantil sobre la modalidad no escolarizada a la todalidad de alumnos que cursaron sus estudios en dicha modalidad en 2019A, de los cuales contestaron 94 . 
Una de las preguntas fue "Las herramientas de comunicación como chat, correo, asesoría académica permiten establecer una comunicación adecuada entre los integrantes de la comunidad"

Contestaron lo siguiente:

$23 \%$ Totalmente de acuerdo.

$52 \%$ De acuerdo.

16\% En Desacuerdo.

9\% Totalmente en desacuerdo.

La encuesta de percepción estudiantil indica que el $25 \%$ está en desacuerdo en que se establece una comunicación adecuada entre los integrantes de la comunidad a través de las herramientas de comunicación dispuestas en la plataforma.

Otra pregunta señala “La retroalimentación llega a tiempo?"

Contestaron lo siguiente

$29 \%$ Totalmente de acuerdo.

$40 \%$ De acuerdo.

\section{3\% En Desacuerdo.}

7\% Totalmente en desacuerdo.

Así que la encuesta de percepción estudiantil indica que el $30 \%$ está en desacuerdo en que la retroalimentación llega a tiempo.

A continuación, se presenta la estadística correspondiente al tiempo de respuesta al evaluar las actividades enviadas por los estudiantes en la modalidad no escolarizada.

Específicamente, se observa el comportamiento para cada periodo del ciclo escolar, desde 2017B a 2019A. Para ello, se obtuvo el promedio de todos los grupos para cada ciclo escolar, su desviación estándar y la gráfica de dispersión.
El comportamiento para el periodo 2017B es un promedio de 8 días con una desviación estándar de 11 días; por lo cual, la mayoría de los profesores demoran entre 0 y 20 días en calificar (ver Tabla 1).

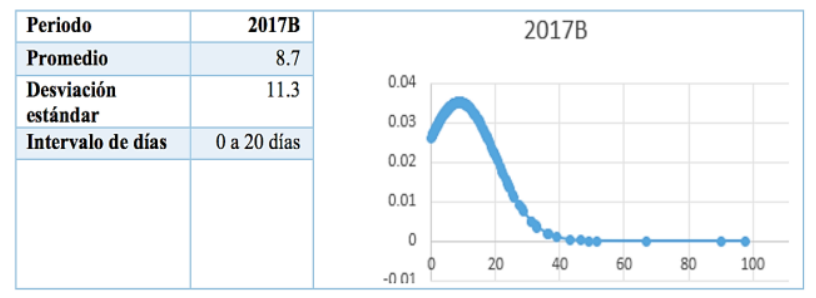

Tabla 1 Tiempo para recibir evaluación de actividades entregadas para 2017B

Fuente: elaboración propia

Para el periodo 2018A es un promedio de 6 días con una desviación estándar de 6 días; por lo cual, la mayoría de los profesores toman de 0 a 13 días en calificar (ver Tabla 2).

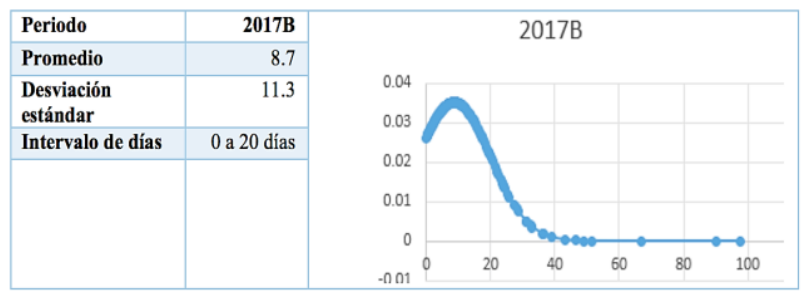

Tabla 2 Tiempo para recibir evaluación de actividades entregadas para $2018^{\mathrm{a}}$

Fuente: elaboración propia

En el periodo 2018B se observa un promedio similar al periodo anterior, de 8 días con una desviación estándar de 9 días; por lo cual, la mayoría de los profesores demoraron entre 0 y 17 días en calificar (Ver Tabla 3).

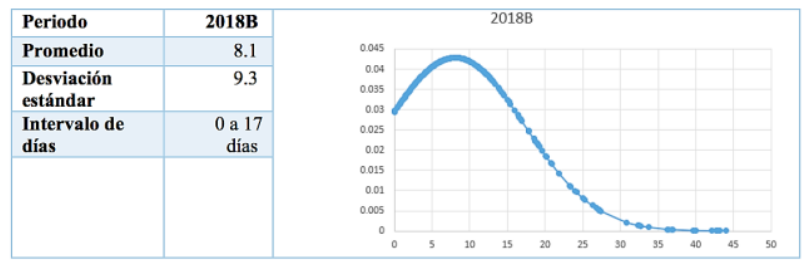

Tabla 3 Tiempo para recibir evaluación de actividades entregadas para 2018B

Fuente: elaboración propia

El comportamiento para el periodo 2019A es un promedio aproximado de 8 días con una desviación estándar de 8 días; por lo cual, la mayoría de los profesores tardan entre 0 y 16 días en calificar. 


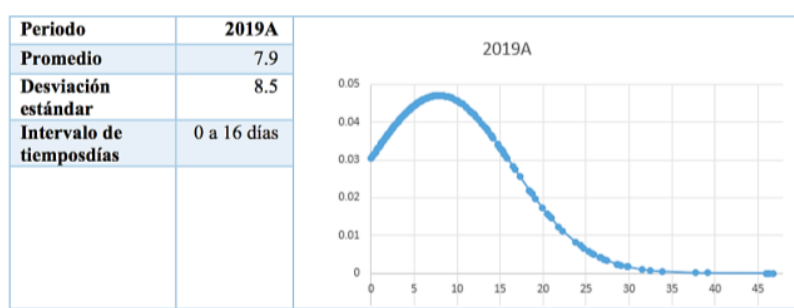

Tabla 4 Tiempo para recibir evaluación de actividades entregadas para $2019^{\mathrm{a}}$

Fuente: elaboración propia

La tabla siguiente se muestra una comparativa entre periodos

\begin{tabular}{|c|c|c|c|c|}
\hline Periodo & 2017B & $2018 \mathrm{~A}$ & $2018 B$ & $2019 A$ \\
\hline Media & 8.7 & 6.6 & 8.1 & 7.9 \\
\hline Desviación estándar & 11.3 & 6.4 & 9.3 & 8.5 \\
\hline Rango de días & $0-20$ & $0-13$ & $1-17$ & $0-16$ \\
\hline
\end{tabular}

Tabla 5

Los días que ocupa un profesor en modalidad no escolarizada (sistema a distancia) para asignar la evaluación a las actividades entregadas por los alumnos es de 6 a 8 días en promedio, con un rango de 0 a 20 días. Al analizar los datos juntos obtenemos un promedio de 7.83 días con una desviación estándar de 9.14. presentando un rango de 0 a 16 días que tarda la mayoría de los maestros en calificar las evidencias, bajo un análisis de datos transversal de 2017B a 2019A.

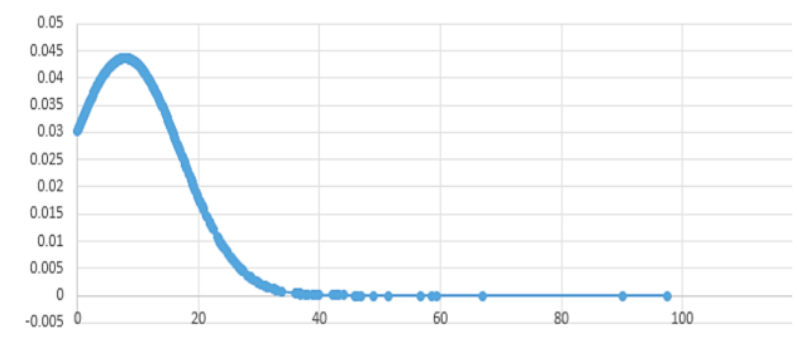

Figura 3 Tiempo para recibir evaluación de actividades entregadas de 2017B a 2019A

Fuente: elaboración propia

También se puede observar en la Figura 3 que a pesar de que las actividades estén asignadas y los alumnos las hayan realizado y entregado a tiempo, algunos profesores no las retroalimentan ni las evalúan.

De acuerdo con la descripción de puesto del Profesor del Sistema a Distancia de la UAEM se tiene un ideal de evaluar las actividades y productos del estudiante dentro de las 72 horas; es decir, en 3 días de haber entregado la evidencia.
El diagnóstico indica que hay una diferencia en promedio de 6 días entre el ideal y el real

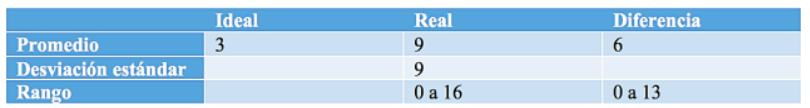

Tabla 6

Las condiciones que actualmente tienen los profesores son:

- Pago quincenal independiente de su desempeño académico.

- Los actores que lo acompañan en su grupo son:

- Coordinador de programa de estudio, quien es integrante en la totalidad de los grupos del programa de estudios en la plataforma SEDUCA y no lleva un seguimiento puntual para cada grupo, además de tener algún otro encargo administrativo.

- Asesor DECyD quien es integrante en la totalidad de los grupos de varios programas de estudios en la plataforma y lleva un seguimiento del desempeño docente que entrega al coordinador del programa de estudio a nivel informativo.

- Tiene libertad de cátedra al asignar las actividades propuestas para el contenido temático; así como la creación de nuevas actividades y asignación de fechas. Éstas últimas en fechas que comprende el periodo lectivo.

En resumen, podemos mencionar la existencia de profesores que:

1. No califican las evidencias de los estudiantes dentro del periodo de tiempo establecido.

2. No responden a las dudas académicas que los estudiantes manifiestan en nuestra plataforma educativa SEDUCA por correo, sección de dudas o chat, dentro del periodo de tiempo establecido. 
3. En el caso de que las actividades estén asignadas y los alumnos las hayan realizado y entregado a tiempo, algunos profesores no las retroalimentan ni las evalúan, incluso cuando los periodos de evaluación están muy avanzados o han concluido.

Tales condiciones pueden dar como resultado que los estudiantes se sientan solos, problemática señalada de manera frecuente como uno de los motivos del alto del abandono escolar en la modalidad a distancia. $\mathrm{O}$ que se vean afectados en su promedio cuando los periodos de asignación de calificaciones en ordinario, extraordinario y título no se atendieron adecuadamente. Sin mencionar el tema de que los estudiantes no logren alcanzar los objetivos establecidos en los programas de estudio de cada UA, lo que, irremediablemente, les afectará en la transición a lo largo del plan de estudios. Se proponen algunas acciones correctivas con la finalidad de disminuir la brecha entre el escenario ideal y el real, entre las que destacan:

- Establecer un actor que dé seguimiento a un número de grupos reducido; de manera que esté en contacto con el docente para dar seguimiento al desempeño y esté atento a las solicitudes de los alumnos.

- Establecer como indicador de pago quincenal el cumplimiento del desempeño docente $o$ en su defecto establecer un esquema de descuento por incumplimiento.

\section{Conclusiones}

Es preciso reflexionar después de tanto engreimiento en torno del verdadero compromiso y responsabilidad del maestro desde sus formas de interpretación de sí mismo y de su mundo fácilmente entendible siempre y cuando se pare uno frente al grupo, que el responsable de planear actividades siempre detrás de su confortable y flamante escritorio entre al campo de batalla en las aulas, para que le permitan al maestro actuar con sentido ético frente a su práctica y tomar conciencia de la influencia de sus propios comportamientos, metas y logros en la formación del futuro individuo funcional.
Para ello, es conveniente preguntar por sus inquietudes laborales, educativas, de preparación continua y permanente; sobre el sentido, significado y finalidad de su quehacer ante la sociedad y los futuros ciudadanos que se encuentran actualmente en su taller de modelado, y sobre cuál es el camino por seguir desde su práctica educativa.

Es provocativo pensar que este país siendo uno de los más ricos del mundo solo tenga tres principales pilares económicos y dos de ellos sean ilegales el primero la piratería discos, lociones, ropa, artículos de belleza. Bebidas alcohólicas improvisadas, películas, software, electrodomésticos son ofertados en el mercado a un precio mucho menor al encontrado en tiendas establecidas, lógicamente la demanda no se hace esperar sin importar si son robados o de dudosa calidad. El siguiente son las divisas extranjeras que paisanos inmigrantes, mojados, indocumentados, ilegales o como se le quiera llamar huyendo de la miseria y falta de trabajo ofertan su fuerza laboral en un país donde se les humilla y maltrata, así un porcentaje mayoritario del sueldo percibido lo mandan a sus familias para una mejora en su calidad de vida. Siendo el petróleo nuestro último el pilar económico que si no es ilegal ya ha sido ofertado al mejor postor internacional.

El fin de la educación es privatizarla, pero esto no se dice, si los padres de familia no quieren dar una cooperación al inicio del ciclo escolar, debería de preocuparse porque después tendrán que pagar por que sus hijos reciban educación, ahora les toca a ellos pedir que evalúen a los profesores más tarde los veremos haciendo marchas porque les apoyen con para pagar las colegiaturas.

Enfrentar todos estos retos no es fácil para los docentes, pero tampoco es imposible los ambientes por lo que concluimos con algunas consideraciones para reformar la educación aquí en México, nuestro país, desde las políticas educativas, los sistemas pedagógicos y la profesionalización docente, como acertadamente dice Edgar Morín (2008) "la reforma de la enseñanza debe conducir a la reforma del pensamiento y la reforma del pensamiento debe conducir a la reforma de la enseñanza" lo que permitirá lograr verdaderamente el cambio en la práctica educativa. 
En resumidas palabras, se necesitan docentes que enseñen con paciencia, amor y exigencia, que desarrollen su creatividad y la de sus alumnos. Paciencia para construir y de construir, amor por sus alumnos y hacia lo que hacen, exigencia para dar lo mejor de sí mismos y para desarrollar la disciplina en sus alumnos y lograr que ellos den más de lo que se les pide. Esforzarse al máximo para formar ciudadanos capaces de transformar su futuro trabajando desde su presente.

\section{Referencias}

Abreu, O. (2014). La dinámica sociedad, universidad, enseñanza-aprendizaje de la historia.

ANUIES. (2017). Programa indicativo para el desarrollo de la educación superior a distancia en México 2024. México: SEP: SINED.

ANUIES-SINED. (2017). Diagnóstico de la Educación Superior a Distancia (1a ed.). México.

Arnaz. José. (2010). Planificación Curricular. México. Trillas.

Domínguez Granda, J.B. (2013). La educación a distancia en el Perú. Hacia la convergencia de las modalidades educativas. Primera edición. Lima.

Dorfsman, M. (2013). La profesión docente en contextos de cambio: El docente global en la sociedad de la información. Recuperado de http://www.um.es/ead/reddusc/6

Fullan, Michel (2002). Los nuevos significados del cambio en la educación, Barcelona, Ed. Octaedro

García Aretio, L. (2011). Perspectivas teóricas de la educación a distancia y virtual. Revista española de pedagogía, (249). pp. 255-271.

García Aretio, L. (2014). Bases, mediaciones y futuro de la educación a distancia en la sociedad digital. Revista de Pensamiento, Tecnología y Sociedad, (98). p.318.

García Aretio, L. (2014). Bases, mediaciones y futuro de la educación a distancia en la sociedad digital. Madrid: Editorial Síntesis.
Huberman, M. (1988), Compromisos con el cambio educativo a través de todo ejercicio docente, Barcelona Ed. Laila

INEE, (2013). La Evaluación de la Educación.México,Ed.INEE

Tejada, J. (1998). Los agentes de la innovación en los Centros Educativos (profesores, directivos y asesores)". Granada, Ed. Aula Santilla

Universidad Autónoma del Estado de México. (2007). Estatuto Universitario. Toluca, México, México: UAEM. 